\title{
Giant isotope effect and spin state transition induced by oxygen isotope exchange in $\left(\mathrm{Pr}_{1-x} \mathrm{Sm}_{x}\right)_{0.7} \mathrm{Ca}_{0.3} \mathrm{CoO}_{3}$
}

\author{
G. Y. Wang, X. H. Chen $⿴$ and T. Wu, G. Wu, X. G. Luo and C. H. Wang \\ Hefei National Laboratory for Physical Science at Microscale and Department of Physics, \\ University of Science and Technology of China, Hefei, \\ Anhui 230026, People's Republic of China
}

(Dated: September 18, 2018)

\begin{abstract}
We systematically investigate effect of oxygen isotope in $\left(\mathrm{Pr}_{1-x} \mathrm{Sm}_{x}\right)_{0.7} \mathrm{Ca}_{0.3} \mathrm{CoO}_{3}$ which shows a crossover with $\mathrm{x}$ from ferromagnetic metal to the insulator with spin-state transition. A striking feature is that effect of oxygen isotope on the ferromagnetic transition is negligible in the metallic phase, while replacing ${ }^{16} \mathrm{O}$ with ${ }^{18} \mathrm{O}$ leads to a giant up-shift of the spin-state transition temperature $\left(T_{s}\right)$ in the insulating phase, especially $T_{s}$ shifts from 36 to $54 \mathrm{~K}$ with isotope component $\alpha_{S}=-4.7$ for the sample with $\mathrm{x}=0.175$. A metal-insulator transition is induced by oxygen isotope exchange in the sample $\mathrm{x}=0.172$ being close to the insulating phase. The contrasting behaviors observed in the two phases can be well explained by occurrence of static Jahn-Teller distortions in the insulating phase, while absence of them in the metallic phase.

PACS numbers: 75.30.-m, 71.38.-k, 31.30.Gs
\end{abstract}

Cobaltites with $\mathrm{CoO}_{6}$ octahedra have recently attracted much attention because they exhibit various intriguing physical properties as magnetoresistance, 1] enormous Hall effect, 2] superconductivity, [3] and large thermoelectric effect. 4] Provskite-related cobalt oxides show their unique feature: temperature-induced spin state transition (SST). [5, 6] Such spin state transition, which is extremely sensitive to composition and pressure, [] is closely connected with electrical conductivity. The $\mathrm{Co}^{3+}$ ion, which contains six $3 \mathrm{~d}$ electrons, often exhibits a SST from the low spin (LS, $t_{2 g}^{6}$ ) ground state to the intermediate spin (IS, $t_{2 g}^{5} e_{g}^{1}$ ) or to the high spin (HS, $t_{2 g}^{4} e_{g}^{2}$ ) state with increasing temperature. The most important character of the SST in perovskite-type cobalt oxides is often accompanied by the metal-insulator transition (MIT).

Experimental and theoretical works indicated that the spin-state transition in cobaltites is from LS to IS below room temperature. [6, 8, 9, 10] The IS state is expected to be strong Jahn-Teller (JT) active due to the partially filled $e_{g}$ level, creating monoclinic distortions of $\mathrm{CoO}_{6}$ octahedra. Actually, elastic and inelastic neutron scattering showed that spin activation of $\mathrm{Co}^{+3}$ ions in $\mathrm{La}_{1-x} \mathrm{Sr}_{x} \mathrm{CoO}_{3}$ system induces local static JT distortions in the paramagnetic insulating phase for all $\mathrm{x}$, while the static JT distortions are absent in the ferromagnetic metal phase. 11] Understanding the characteristics of Jahn-Teller instability can help elucidate the nature of metal-insulator transition, magnetoresistance, and even superconductivity. 12] The IS-JT state is stabilized with doping in the insulating phase for perovskite cobalt oxides. 6, 13] However, charge dynamics interfere with orbital JT ordering resulting in a new state and the JT distortions can lose their long-range coherency with increasing charge mobility in the ferromagnetic metal phase. JT interactions in perovskite oxides often result in quite intriguing physical phenomena through the coupling between orbital and spin degrees of freedom with lattice. 14 Coupling of JT modes to the lattice results in formation of small JT polarons, so that coupling of the charge carrier to the JT lattice distortions leads to a giant oxygen isotope effect, and the JT distortions play an essential role in $\mathrm{La}_{1-x} \mathrm{Ca} \mathrm{a}_{x} \mathrm{MnO}_{3+y}$. 15] Especially, a metal-insulator transition was induced by oxygen isotope exchange in $\mathrm{La}_{0.175} \mathrm{Pr}_{0.525} \mathrm{Ca}_{0.3} \mathrm{MnO}_{3}$. [16] To investigate JT physics and its role played in cobaltites, here we systematically study effect of oxygen isotope in perovskite $\left(\mathrm{Pr}_{1-x} \mathrm{Sm}_{x}\right)_{0.7} \mathrm{Ca}_{0.3} \mathrm{CoO}_{3}$ system with different $\mathrm{x}$. This system is suitable to study the JT physics by oxygen isotope effect since it shows a crossover at $\mathrm{x} \sim 0.175$ from a ferromagnetic metal phase to an insulating phase with SST with increasing x. 17, 18] Therefore, it is expected that effect of oxygen isotope should be quite different in the ferromagnetic metal phase from in the insulating phase because the static JT distortions are observed in the insulating phase, while absent in the metallic phase.

Polycrystalline samples $\left(\mathrm{Pr}_{1-x} \mathrm{Sm}_{x}\right)_{0.7} \mathrm{Ca}_{0.3} \mathrm{CoO}_{3}$ with different $\mathrm{x}$ were prepared by conventional solidstate reaction. Proper molar ratios of $\mathrm{Pr}_{6} \mathrm{O}_{11}, \mathrm{Sm}_{2} \mathrm{O}_{3}$, $\mathrm{CaCO}_{3}$ and $\mathrm{Co}_{3} \mathrm{O}_{4}$ were mixed, well ground and then calcined at $1000{ }^{\circ} \mathrm{C}$ and $1100{ }^{\circ} \mathrm{C}$ for $24 \mathrm{~h}$ with intermittent grinding. The pellets pressed from the powder were sintered at $1200{ }^{\circ} \mathrm{C}$ in the flowing oxygen for 48 h. All samples were characterized by X-ray diffraction and found to be single phase. One pellet for the samples with different $\mathrm{x}$ obtained above was cut into two pieces for oxygen-isotope diffusion. The two pieces for each composition were put into an alumina boat which were sealed in a quartz tube filled with oxygen pressure 
of 1 bar (one for ${ }^{16} \mathrm{O}$ and another for ${ }^{18} \mathrm{O}$ ) mounted in a furnace, respectively. The quartz tubes formed parts of two identical closed loops. They were heated at $1000{ }^{\circ} \mathrm{C}$ for $48 \mathrm{~h}$ and then slowly cooled to room temperature. The obtained samples were re-examined by X-ray diffraction to confirm them single phase. The oxygen-isotope enrichment is determined from the weight changes of both ${ }^{16} \mathrm{O}$ and ${ }^{18} \mathrm{O}$ samples. The ${ }^{18} \mathrm{O}$ samples have about $70 \%{ }^{18} \mathrm{O}$ and $30 \%{ }^{16} \mathrm{O}$. To make sure the isotope exchange effect, back-exchange of ${ }^{18} \mathrm{O}$ sample by ${ }^{16} O$ was carried out in the same way and the weight change showed a complete back-exchange. Magnetic susceptibility measurements were performed with a SQUID magnetometer in a magnetic field of 1000 Oe. Resistance measurements were performed using the ac four-probe method with an ac resistance bridge system (Linear Research Inc. LR-700P).

Figure 1 shows temperature dependence of resistivity for the samples $\left(\mathrm{Pr}_{1-x} S m_{x}\right)_{0.7} \mathrm{Ca}_{0.3} \mathrm{CoO}_{3}$ with $\mathrm{x}=0.175$, $0.20,0.25$ and 0.30 treated in ${ }^{16} O$ and ${ }^{18} O$, respectively. The samples $\left(\mathrm{Pr}_{1-x} \mathrm{Sm}_{x}\right)_{0.7} \mathrm{Ca}_{0.3} \mathrm{CoO}_{3}$ with various $\mathrm{x}$ treated in ${ }^{16} \mathrm{O}$ shows a very weak semiconductive behavior at the high temperatures, while a sharp metalinsulator transition is observed at certain temperatures $\left(T_{S}\right)$ for different $\mathrm{x}$ with decreasing temperature. $T_{S}$ increases with increasing $\mathrm{x}$. As shown in Fig.1, substitution of ${ }^{18} \mathrm{O}$ for ${ }^{16} \mathrm{O}$ leads to an enhancement of $T_{S}$ for all samples. The up-shift of $T_{S}$ decreases with increasing $\mathrm{x}$. A giant isotope shift is observed in the sample with $\mathrm{x}=0.175$. $T_{S}$ shifts from 36 to $54 \mathrm{~K}$ after replacing of ${ }^{16} \mathrm{O}$ by ${ }^{18} \mathrm{O}$. The oxygen isotope exponent $\alpha_{s}=-d \ln T_{S} / d \ln M_{O}$ (where $M_{O}$ is the oxygen isotope mass) is about -4.7 , which is much larger than $\alpha_{c}=0.8$

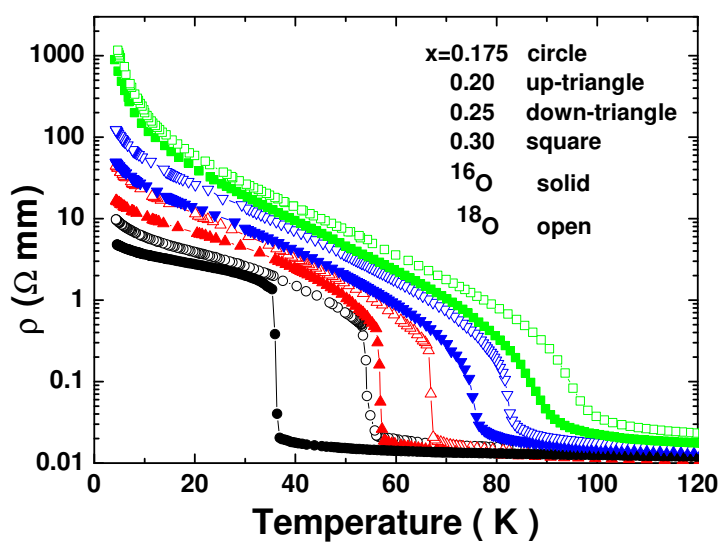

FIG. 1: Temperature dependence of resistivity for the ${ }^{16} \mathrm{O}$ and ${ }^{18} \mathrm{O}$ samples $\left(\mathrm{Pr}_{1-x} \mathrm{Sm}_{x}\right)_{0.7} \mathrm{Ca}_{0.3} \mathrm{CoO}_{3}$ with $\mathrm{x}=0.175$, $0.20,0.25$ and 0.30 . The data denoted by the solid and open symbols are taken from the samples treated in ${ }^{16} \mathrm{O}$ and ${ }^{18} \mathrm{O}$, respectively. observed in $L a_{1-x} C a_{x} M n O_{3+y} \cdot 15$. However, the upshift of $T_{S}$ is about $7 \mathrm{~K}$ for the sample with $\mathrm{x}=0.30$ and the isotope component $\alpha_{s}$ is about -0.8. To make sure that the effect arises from the oxygen isotope exchange, we carried out the isotope back-exchange. The transport properties completely return to that observed in the samples previously treated in ${ }^{16} \mathrm{O}$ and ${ }^{18} \mathrm{O}$ after the isotope back-exchange, respectively. Such metal-insulator transition has been believed to arise from the spin-state transition. Therefore, the susceptibility was measured for the samples above.

Temperature dependence of susceptibility measured in the field-cooled process with magnetic field of 1000 Oe is shown in Fig.2 for the same samples used in resistivity measurements. All samples show a sharp SST from IS state to LS state with decreasing temperature, respectively. $T_{s}$ increases with increasing $\mathrm{x}$. Replacing of ${ }^{16} \mathrm{O}$ with ${ }^{18} \mathrm{O}$ makes $T_{s}$ shift to high-temperature. This is consistent with metal-insulator transition observed in resistivity as shown in Fig.1. It should be pointed out that $T_{s}$ is exactly the same as that of metal-insulator transition observed in Fig.1. The sample with $\mathrm{x}=0.175$ treated in ${ }^{16} \mathrm{O}$ and ${ }^{18} \mathrm{O}$ show a sharp SST from IS state to LS state with decreasing temperature at 36 and $54 \mathrm{~K}$, respectively. It further supports that the metal-insulator transition arises from the SST. It has been noted that there exists a thermal hysteresis for the phase transition. Therefore, all measurements were carried out in the cooled process to keep the consistency.

Figure 3 shows temperature dependences of resistivity and susceptibility for the ${ }^{16} \mathrm{O}$ and ${ }^{18} \mathrm{O}$ samples $\left(\mathrm{Pr}_{1-x} \mathrm{Sm}_{x}\right)_{0.7} \mathrm{Ca}_{0.3} \mathrm{CoO}_{3}$ with $\mathrm{x}=0.172$. The sample treated in ${ }^{16} \mathrm{O}$ shows a very weak semiconductive be-

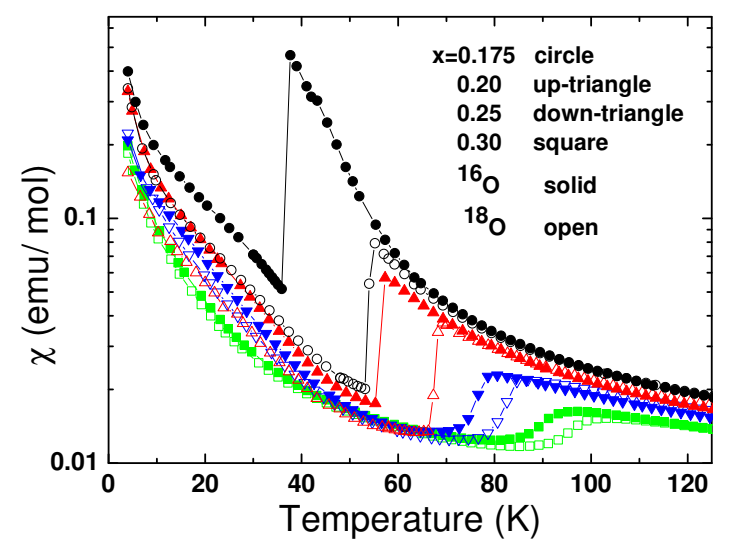

FIG. 2: Temperature dependence of susceptibility for the ${ }^{16} \mathrm{O}$ and ${ }^{18} \mathrm{O}$ samples $\left(\mathrm{Pr}_{1-x} \mathrm{Sm}_{x}\right)_{0.7} \mathrm{Ca}_{0.3} \mathrm{CoO}_{3}$ with $\mathrm{x}=0.175$, $0.20,0.25$ and 0.30 . The data denoted by the solid and open symbols are taken from the samples treated in ${ }^{16} \mathrm{O}$ and ${ }^{18} \mathrm{O}$, respectively. 


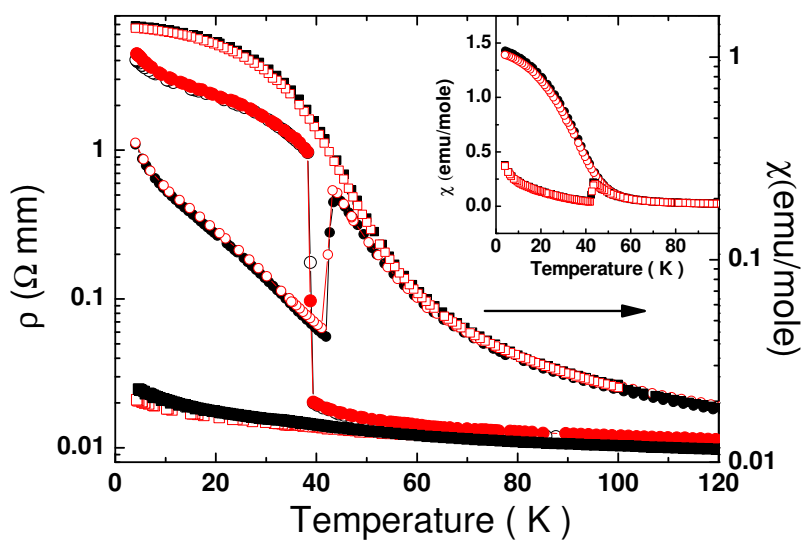

FIG. 3: Temperature dependences of resistivity and susceptibility for the sample $\left(\mathrm{Pr}_{1-x} \mathrm{Sm}_{x}\right)_{0.7} \mathrm{Ca}_{0.3} \mathrm{CoO}_{3}$ with $\mathrm{x}=0.172$ (solid squares for the ${ }^{16} \mathrm{O}$ sample; solid circles for ${ }^{18} \mathrm{O}$ sample). The data denoted by the open symbols were obtained after the oxygen isotope back-exchange $\left({ }^{16} \mathrm{O} \rightarrow{ }^{18} \mathrm{O}\right.$ and $\left.{ }^{18} \mathrm{O} \rightarrow{ }^{16} \mathrm{O}\right)$. The reversibility of the metal-insulator transition induced by oxygen isotope exchange is clear.

havior at the low temperatures and no phase transition, while a sharp metal-insulator transition is observed at about $39 \mathrm{~K}$ after the treatment in ${ }^{18} \mathrm{O}$. It suggests that a metal-insulator transition is induced by oxygen isotope exchange. To make sure that the effect arises from the oxygen isotope exchange, we carried out the isotope backexchange. As shown in Fig.3, the transport properties completely return to that observed in the samples previously treated in ${ }^{16} \mathrm{O}$ and ${ }^{18} \mathrm{O}$ after the isotope backexchange, respectively. The remarkable feature is that the metal-insulator transition disappears after the subsequent treatment in ${ }^{16} \mathrm{O}$, while shows up after the subsequent treatment in ${ }^{18} \mathrm{O}$. It definitely indicates that the metal-insulator transition is induced by replacing ${ }^{16} \mathrm{O}$ with ${ }^{18} \mathrm{O}$.

As shown in Fig.3, a striking feature for susceptibility is that the sample $\left(\mathrm{Pr}_{1-x} \mathrm{Sm}_{x}\right)_{0.7} \mathrm{Ca}_{0.3} \mathrm{CoO}_{3}$ with $\mathrm{x}=0.172$ treated in ${ }^{16} \mathrm{O}$ shows a ferromagnetic transition at about $60 \mathrm{~K}$, while the ferromagnetic transition disappears and a sharp spin-state transition at about $42 \mathrm{~K}$ is induced after replacing of ${ }^{16} \mathrm{O}$ with ${ }^{18} \mathrm{O}$. To clearly show a ferromagnetic transition, the same data are plotted with linear vertical axis in the inset of Fig.3. It indicates that the replacement of ${ }^{16} O$ with ${ }^{18} O$ suppresses the ferromagnetic phase, while induces a SST in the sample with $\mathrm{x}=0.172$. It suggests that there exists a competition between the ferromagnetic transition and SST. This result is consistent with that observed in resistivity measurements. It should be pointed out that the transition temperature in susceptibility is about $3 \mathrm{~K}$ higher than that in resistivity, in contrast to the case for samples with $x \geq 0.175$. The isotope back-exchange results fur- ther confirm that the behavior observed in Fig.3 is due to oxygen isotope exchange. Beyond the expectation, a phase transition from ferromagnetic metal to an insulator with spin-state transition is induced by replacing replacing ${ }^{16} \mathrm{O}$ with ${ }^{18} \mathrm{O}$.

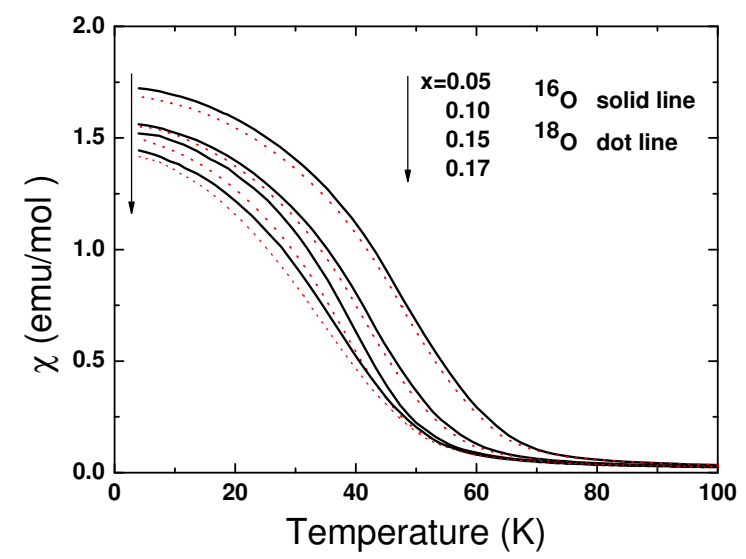

FIG. 4: Temperature dependence of susceptibility for the ${ }^{16} \mathrm{O}$ and ${ }^{18} \mathrm{O}$ samples $\left(\mathrm{Pr}_{1-x} \mathrm{Sm}_{x}\right)_{0.7} \mathrm{Ca}_{0.3} \mathrm{CoO}_{3}$ with $\mathrm{x}=0.05$, $0.10,0.15$ and 0.17 : solid lines for ${ }^{16} \mathrm{O}$ samples; dot lines for ${ }^{18} \mathrm{O}$ samples.

To further study the JT physics by oxygen isotope effect in the ferromagnetic metal phase, the susceptibility is systematically studied on the ${ }^{16} \mathrm{O}$ and ${ }^{18} \mathrm{O}$ samples $\left(\mathrm{Pr}_{1-x} \mathrm{Sm}_{x}\right)_{0.7} \mathrm{Ca}_{0.3} \mathrm{CoO}_{3}$ with $\mathrm{x}=0.05,0.10,0.15$ and 0.17 . Temperature dependence of susceptibility for these samples are shown in Fig.4. Actually, substitution of ${ }^{18} \mathrm{O}$ for ${ }^{16} O$ suppresses the ferromagnetic transition and leads to a down-shift of the Curie temperature $\left(T_{c}\right)$, being similar to that observed in mamganites although the downshift of $T_{c}$ is much less relative to $L a_{1-x} C a_{x} M n O_{3+y}$. 15. The down-shift of $T_{c}$ slightly increases with increasing x. However, the shift of $T_{c}$ is very small compared to that of $T_{S}$. It suggests that the effect of oxygen isotope on ferromagnetic transition is negligible.

In order to compare the effect of oxygen isotope in the ferromagnetic metallic phase and in the insulating phase with spin-state transition, the isotope components $\alpha_{s}$ for SST and $\alpha_{c}$ for ferromagnetic transition as a function of $\mathrm{x}$ and the average ionic radius of $\mathrm{A}$ site are shown in Fig.5. The isotope component $\alpha_{s}$ decreases with increasing Sm content $\mathrm{x}$ in $\left(\mathrm{Pr}_{1-x} \mathrm{Sm}_{x}\right)_{0.7} \mathrm{Ca}_{0.3} \mathrm{CoO}_{3}$ system. As observed in Fig.1 and Fig.2, $T_{S}$ increases with increasing $\mathrm{x}$, while its isotope shift $\Delta T_{s}$ decreases. Therefore, it leads to a decrease of the isotope component $\alpha_{s}$ with increasing $\mathrm{x}$. Substitution of ${ }^{16} \mathrm{O}$ by ${ }^{18} \mathrm{O}$ leads to a decrease in the Curie temperature $\left(T_{c}\right)$ although its oxygen isotope shift is negligible. Its isotope component $\alpha_{c}$ is positive and increases with increasing $\mathrm{x}$, in contrast to the $\alpha_{s}$. It further confirms that there exists a 


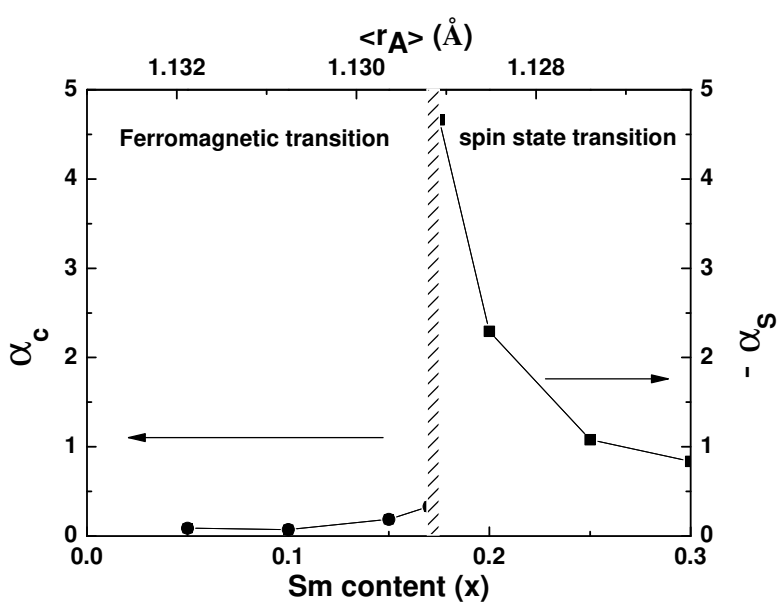

FIG. 5: The oxygen isotope exponent $\alpha_{c}$ and $\alpha_{s}$ as a function of Sm content $\mathrm{x}$ and the average ionic radius $\left\langle r_{A}\right\rangle$ of A site $\left(\mathrm{Pr}_{1-x} \mathrm{Sm}_{x}\right)_{0.7} \mathrm{Ca}_{0.3}$ in $\left(\mathrm{Pr}_{1-x} \mathrm{Sm}_{x}\right)_{0.7} \mathrm{Ca}_{0.3} \mathrm{CoO}_{3}$ system.

competition between ferromagnetic transition and SST. As shown in Fig.5, a maximum isotope component $\alpha$ is observed in the boundary between ferromagnetic metal and insulating phases. A intriguing phenomenon is that the substitution of ${ }^{16} \mathrm{O}$ by ${ }^{18} \mathrm{O}$ leads to a disappearance of ferromagnetic transition and induces the SST accompanied by the metal-insulator transition in the sample $\left(\mathrm{Pr}_{0.828} \mathrm{Sm}_{0.172}\right)_{0.7} \mathrm{Ca}_{0.3} \mathrm{CoO}_{3}$ which is in the ferromagnetic metal phase close to the crossover boundary.

In the insulating regime $(x>0.175)$, the up-shift of $T_{S}$ induced by oxygen isotope exchange can be explained to originate from that substitution of ${ }^{16} \mathrm{O}$ by ${ }^{18} \mathrm{O}$ leads to a decrease in frequency of phonon, and consequently enhances effective mass of electron $\left(m^{*}\right)$ through strong electron-phonon coupling. The enhancement of $m^{*}$ leads to a decrease of bandwidth $\mathrm{W}$, resulting in an increase of energy difference $\Delta E$ between IS and LS states and consequently an up-shift of $T_{s}$. The increase of Smdoping leads to a decrease in the ionic radius of $\mathrm{A}$ site $\left(\mathrm{Pr}_{1-x} \mathrm{Sm}_{x}\right)_{0.7} \mathrm{Ca}_{0.3}$ in the $\left(\mathrm{Pr}_{1-x} \mathrm{Sm}_{x}\right)_{0.7} \mathrm{Ca}_{0.3} \mathrm{CoO}_{3}$ system, so that volume of the $\mathrm{CoO}_{6}$ octahedra decreases. The decrease in volume of the $\mathrm{CoO}_{6}$ octahedra results in an increase of crystal field splitting energy $\Delta_{C}$ and $\Delta E$, causing an increase in $T_{S}$. Therefore, decrease of the isotope component $\alpha_{s}$ with increasing x could be due to the increase of $T_{s}$. In contrast to the giant oxygen isotope effect in the insulating phase, a negligible oxygen isotope effect on $T_{c}$ is observed in the ferromagnetic metal regime. It has been reported that substitution of ${ }^{16} \mathrm{O}$ by ${ }^{18} \mathrm{O}$ leads to a giant down-shift of Curie temperature in $L a_{1-x} C a_{x} M n O_{3+y}$. 115] Such giant oxygen isotope shift was explained by the JT polaron formed due to strong Jahn-Teller effect. 15] Recently, elastic and inelastic neutron scattering showed that spin activation of $\mathrm{Co}^{+3}$ ions in perovskite cobalt oxides induces local static JT distortions in the insulating phase, while the static JT dis- tortions are absent in the ferromagnetic metal phase. 11] This observation explained that the giant oxygen isotope effect is observed in the insulating phase, while negligible in the ferromagnetic metal phase well. The lattice distortions from the JT effect with strong electron-phonon coupling should be responsible for the giant isotope effect in the insulating phase.

In conclusion, oxygen isotope effect is systematically studied in $\left(\mathrm{Pr}_{1-x} \mathrm{Sm}_{x}\right)_{0.7} \mathrm{Ca}_{0.3} \mathrm{CoO}_{3}$ with a crossover at $\mathrm{x} \sim 01.75$ from a ferromagnetic metal to an insulating phase with SST. A giant oxygen isotope effect is observed in the insulating phase with an isotope component $\alpha_{S}=-4.7$ for the sample with $\mathrm{x}=0.175$ which is significantly larger than that found for any magnetic or electronic phase transition in other oxides, while a negligible oxygen isotope effect occurs in the ferromagnetic metal phase. Beyond the expectation, substitution of ${ }^{16} \mathrm{O}$ by ${ }^{18} \mathrm{O}$ leads to a phase transition from a ferromagnetic metal to an insulator in the sample with $\mathrm{x}=0.172$ which lies in the ferromagnetic metal phase close to the crossover boundary. These contrasting behaviors are in support of the observation that static JT distortions occurs in the insulating phase, while are absent in the ferromagnetic metal phase. 11] It provides a direct demonstration of the important of lattice vibrations in these materials.

Acknowledgement: This work is supported by the Nature Science Foundation of China, and by the Ministry of Science and Technology of China (973 project No:2006CB601001), and by the Knowledge Innovation Project of Chinese Academy of Sciences.

Corresponding author; Electronic address: chenxh@ustc.edu.cn

[1] G. Briceno, H. Y. Chang, S. D. Sun, P. G. Schultz and X. D. Xiang, Science 270, 273(1995).

[2] A. V. Samoilov, G. Beach, C. C. Fu, N. C. Yeh, and R. P. Vasquez, Phys. Rev. B 57, R14032(1998).

[3] K. Takada et al., Nature 422, 53(2003).

4. T. Terasaki et al., Phys. Rev. B 56, R12685(1997).

5. M. A. Senaris-Rodriguez and J. B. Goodenough, J. Solid State Chem. 116, 224(1995).

6] S. Tsubouchi et al., Phys. Rev. B 66, 052418(2002).

7. K. Asai et al., J. Phys. Soc. Jpn 66, 967(1997).

[8] S. Yamaguchi, Y. Okimoto and Y. Tokura, Phys. Rev. B55, R8666(1997).

[9] T. Saitoh et al., Phys. Rev. B56, 1290(1997)

[10] M. A. Korotin et al., Phys. Rev. B54, 5309(1996).

11] D. Louca and J. L. Sarrao, Phys. Rev. Lett. 91, 155501(2003).

[12] I. B. Bersuker, Chem. Rev. 101, 1067(2001).

13. D. Louca et al., Phys. Rev. B 60, R10378(1999).

14. J. B. Goodenough, Mater. Res. Bull. 6, 967(1971).

15 G. M. Zhao, K. Conder, H. Keller, and K. A. Mller, Nature 381, 676(1996).

[16] N. A. Babushkina et al., Nature 391, 159(1998).

17. T. Fujita et al., J. Phys. Soc. Jpn 73, 1987(2004).

18] T. Fujita et al., J. Phys. Soc. Jpn 74, 2294(2005). 